\title{
TASAS DE NATALIDAD Y TASAS DE AHORRO
}

\author{
Nathaniel H. LeFF \\ Universidad Columbia
}

EN LA MAYORÍA DE LA DISCUSIONES acerca de los determinantes de las tasas de ahorro interno agregado $(S / Y)$ en los países subdesarrollados ha tenido papel importante el nivel del ingreso per capita. Este punto de vista está apoyado en la observación de que las tasas de ahorro agregado son generalmente más altas en los países ricos que en los pobres, y ha recibido algo de apoyo de los estudios estadísticos de tasas de ahorro internacionales.1 Estos estudios, sin embargo, dan lugar a una duda importante. Los resultados de sección transversal sugieren que con niveles crecientes de ingreso, en países individuales, las tasas de ahorro agregado deberían crecer igualmente. ${ }^{2}$ Esto no ha sido respaldado, sin embargo, por las series de tiempo de la mayoría de los países. El ingreso per capita ha crecido a tasas anuales entre 1 y $2.5 \%$ en la mayoría de los países subdesarrollados durante los pasados 15 años, y muchos de los cambios sociales y económicos que se esperaba elevaran las tasas de ahorro agregado han ocurrido de hecho; no obstante, las tasas de ahorro generalmente no han mostrado tendencia ascendente. ${ }^{3}$

Este trabajo considera una hipótesis que puede explicar ese fenómeno, ${ }^{4}$ y presenta resultados estadísticos para probarla. En seguida se examinan algunas implicaciones analíticas y de política.

I Véase, por ejemplo, Charles Wolf, Jr. (51, p. 19); Naciones Unidas (48, pp. $13-$ 18); Luis Landau (28). H. S. Houthakker (24, pp. 220-221) también mostró alguna evidencia de ligera curvilinearidad entre el ingreso y el ahorro interno bruto. El trabajo pionero en esta dirección fue, por supuesto, el de Simón Kuznetz (27). (Nota: la crítica metodológica hecha por Houthakker a Kuznetz por no ponderar sus observaciones con el tamaño de la población no es pertinente en el presente contexto, porque Kuznetz estaba interesado, como el presente estudio, en las tasas de ahorro de países y no de individuos.) [Ver bibliografía al final.]

2 Los resultados de Alan Strout (43, cuadros 3-4) y Hollis B. Chenery y Peter Eckstein (5) también indicaron tasas marginales de ahorro superiores al promedio. Modigliani y Brumber $(35,33)$ y Milton Friedman (21), por supuesto, han sostenido sobre bases teóricas que a largo plazo la relación ingreso/ahorro debe ser constante. Esto se examina más adelante.

3 Véase $(48, \mathrm{pp}, 14-16)$ respecto a datos sobre la evolución de las tasas de ahorro interno en 21 países en desarrollo entre 1953-1955 y 1962-1964. Durante este periodo, sólo las tasas de tres de ellas mostraron aumento apreciable (superior a 3 puntos de porciento), mientras que 8 países tuvieron disminuciones en sus tasas de ahorro interno bruto. En la misma sección aparecen datos sobre la tasa de crecimiento del ingreso en países subdesarrollados durante este período.

4 Este problema es, por supuesto, similar al que surgió en los años cuarenta en Estados Unidos y discutido por Duesenberry (16), Modigliani y Brumberg (34), y Friedman. Sin embargo, tal vez debido a que esa discusión se centra sobre la función consumo (privado), tiende a concentrarse en el ahorro familiar más que 


\section{LAS CONDICIONES DEMOGRÁFICAS Y EL AHORRO}

Una manera lógica de salir del problema sección transversal-series de tiempo es que las ecuaciones de $S / Y$ contra ingreso per capita $(Y / N)$ estuvieran incorrectamente especificadas. La correlación entre $S / Y$ e $Y / N$ puede, al menos en parte, reflejar una conexión entre $S / Y$ y otra variable que no cambió a medida que los niveles de ingreso per capita se elevaron. Las tasas brutas de fecundidad son un candidato obvio para esta "verdadera" variable. Estas tasas, cuyo valor oscila entre 35 y 45 por millar en la mayoría de los países subdesarrollados y entre 17 y 23 en la mayoría de los clesarrollados, guardan alta correlación con los niveles de ingreso per capita, ${ }^{5}$ y por añadidura dichas tasas generalmente no han declinado en el curso del desarrollo de postguerra. ${ }^{B}$

La idea de que los factores demográficos deberian incluirse en el análisis de las tasas de ahorro es también recomendable desde el punto de vista analítico. Un número de economistas ha sugerido por mucho tiempo que, a través de un proceso elaborado en la siguiente sección de este trabajo, las tasas de fecundidad deben guardar correlación inversa con el ahorro potencial de un país. ${ }^{7}$ Esta sugerencia está también apoyada por casos empíricos: Japón, Luxemburgo y Noruega, que son "excepciones" a un modelo ingreso per capita-tasa de ahorros, ${ }^{8}$ se ajustan perfectamente a un enfoque que incluya condíciones demográficas, ya que sus tasas de natalidad son todas bajas con relación a sus niveles de ingreso. Las altas tasas de ahorro de los países socialistas de Europa Oriental también se ajustan a este patrón. Como lo indica el cuadro 1, las tasas de natalidad en esos países descendieron agudamente durante los años veinte y treinta, y alcanzaron niveles relativamente bajos antes de que los comunistas tomaran el poder. ${ }^{9}$ Estos datos sugieren que las condiciones demográficas favorables pudieron haber sido una razón -en adición a factores de demanda y sus rasgos institucionales especiales- que posibilitó a estos países el lograr tasas de ahorro e inversión relativamente elevadas.

Las consideraciones anteriores exigen con urgencia la inclusión de las condiciones demográficas en los estudios empíricos de-tasas de ahorro agregado y esto se hace más importante en vista de que los estudios econométricos de $S / Y$ no han tenido mucho éxito estadístico.10

en la tasa de ahorro agregado. Como se verá más adelante, las teorías sobre la función consumo citadas pueden ser integradas fácilmente con las hipótesis discutidas en este trabajo.

3 La correlación simple para 74 países es -.71 , calculada con los datos que se examinan más adelante.

6 Véanse los datos disponibles por países en el Demographic Yearbook de las Naciones Unidas (45).

7 Véanse, por ejemplo, Hans W. Singer (40, pp. 80-81); Joseph J. Spengler (41);

Ansley B. Coale y Edgar M. Hoover (10, pp. 22-24).

8 Houthakker, op. cit., p. 217.

- En relación con la temprana reducción de las tasas de fecundidad en Euro pa Oriental, véase (37) y Paul Demény (14).

10 Por ejemplo, Wolf (51) y Landau (28) encuentran coeficiente de determinación múltiple de .2 a .3 en ecuaciones para $S / Y$, en comparación con las obtenidas en ecuaciones de regresión del ahorro per capita sobre el ingreso per capita 
Cuadro 1

Tasas bRUtas de Natalidad EN Países de Europa OrIEntal (Nacidos vivos por millar)

\begin{tabular}{lcccccc}
\hline \multicolumn{1}{c}{ Pals } & $1920-24$ & $1925-29$ & $1930-34$ & $1935-39$ & $1940-44$ & $1955-59$ \\
\hline Bulgaria & 39.6 & 34.2 & 30.3 & 24.1 & 22.1 & 18.7 \\
Checoeslovaquia & 26.8 & 22.9 & 19.7 & 17.1 & 20.8 & 18.5 \\
Alemania Oriental & 23.1 & 19.1 & 16.3 & 19.4 & 17.4 & 16.3 \\
Hungria & 30.2 & 26.6 & 23.2 & 20.1 & 19.3 & 17.8 \\
Polonia & 34.3 & 32.9 & 28.9 & 25.4 & n.d. & 22.1 \\
Rumania & 37.6 & 35.4 & 32.9 & 30.0 & 26.0 & 22.9 \\
Yugoslavia & 35.3 & 33.9 & 33.0 & 27.7 & $n_{.}$d. & 24.8 \\
U.R.S.S. & n.d. & 44.4 & n.d. & 37.6 & 31.4 & 25.3 \\
\hline
\end{tabular}

Fuente: Naciones Unidas, Demographic Yearbook 1965 (Nueva York, 1966).

Al mismo tiempo, aunque las condiciones demográficas han sido sugeridas por algunos autores como determinantes importantes de las tasas de ahorro, la idea no ha estado en el centro de la discusión; y verdaderamente, algunos estudios y textos sobre desarrollo económico casi ni la mencionan. ${ }^{11}$ Una razón de ello es que la hipótesis no ha sido avalada por pruebas empíricas extensas. En consecuencia, debe valer la pena ver si la inclusión de los factores demográficos aumenta estadísticamente nuestra capacidad para explicar el 'comportamiento de los ahorros.

\section{LA HIIPÓTESIS}

La noción de que las altas tasas de natalidad constriñen el ahorro tiene dos componentes. Primero, las teorías demográficas $(8,9)$ indican que una tasa de natalidad prolongada y alta afectará la composición de edades de la población, y colocará un porciento relativamente elevado de ella en los estratos jóvenes. Esta relación está, de hecho, apoyada por la experiencia demográfica reciente..$^{12}$

en las que $R^{2}$ resultó entre .8 y .9 . Por añadidura, nuestro conocimiento sobre el comportamiento de los ahorros puede no ser tan sustancial como podrían sugerir los resultados estadísticos de los estudios sobre el ahorro per capita. Maurice Wilkinson me ha señalado que el elevado valor de $R^{2}$ obtenido en las regresiones de $S / N$ puede ser resultado de un artificio estadístico. Si multiplicamos por $Y / N$ el lado izquierdo de la relación $S / Y=f(Y / N)$ y multiplicamos $S / Y$ por $Y / N$ para obtener $S / N$, el resultado puede ser un alto coeficiente de correlación debido a la presencia de $Y / N$ en ambos lados de la ecuación. Pero la relación estructural subyacente es $S / Y$, para la cual, como se indicó, los resultados estadísticos no han sido muy satisfactorios.

11 Véanse, por ejemplo, los estudios de Morris (56), Wolfe (51), Landau (28), Chenery y Eckstein (5), y C. P. Kindleberger (25), capítulos 5 y 15.

12 Se estimó por mínimos cuadrados una ecuación de regresión, tomando. como variable endógena el cociente de dependencia infantil $D_{1}$ (la proporción de personas de 14 años o menos en el total de la población), y como exógena la tasa de natalidad de 14 años atrás (o la del año más cercano), $B_{14}$. Esta información proviene de Demographic Yearbook, 1965 de las Naciones Unidas (41). La ecuación (1), en la que aparecen entre paréntesis los errores estándar, indica la impor- 
La lógica de una relación inversa entre las razones de dependencia y las tasas de ahorro, a su vez, es como sigue. Como ha sido señalado a menudo, los niños constituyen una pesada carga en gastos que en los esquemas usuales de contabilidad del ingreso nacional se ponen bajo el encabezado que dice consumo. Ya que estos dependientes deben ser mantenidos a algún nivel de vida mínimo, el costo de su manutención puede ser considerado como gasto en consumo fijo y participante de los gastos discrecionales de las familias. ${ }^{13}$ Como contribuyen al consumo y no a la producción, puede esperarse que una alta proporción de dependientes respecto a la población en edad de trabajar imponga restricción al potencial de ahorro de una sociedad. ${ }^{14}$

Dicho ahorro puede concebirse como proveniente de tres fuentes: ahorro privado de los particulares, ahorro de las empresas y el excedente del gobierno en cuenta corriente. En nuestra hipótesis el vínculo entre las razones de dependencia y el ahorro personal bajo es, como acaba de describirse, directo.15 También puede sugerirse una conexión con el ingreso del gobierno -y tal vez excedente- si suponemos que el límite de la carga impositiva es fijado por un mínimo per aapita (convencional) de nivel de vida. De aquí que la carga de consumo impuesta por los niños entre de nuevo como restricción a los impuestos. No hay razón obvia de por qué el cociente de dependencia debiera afectar el ahorro de las empresas, pero debemos reconocer la interdependencia entre las decisiones para ahorrar en los sectores empresarial, familiar y público.18 Además, en países donde la propiedad empresarial no está muy difundida, la frontera entre el ingreso de las empresas y el familiar - y el ahorro - no es clara y definida. Similarmente, en muchos países, las empresas del gobierno juegan papel económico importante, sobre todo en industrias intensivas de capital

tancia de la tasa retardada de natalidad para explicar el cociente de dependencia infantil.

$$
\begin{gathered}
\ln D_{1}=\underset{(.0867)}{1.4654}+\underset{(.0252)}{.6195} \ln B_{14} \\
\bar{R}^{2}=.889 \quad F=510.6
\end{gathered}
$$

13 Puede decirse que esto tiene el mismo espíritu (pero la realidad empirica completamente opuesta) que las decisiones para invertir y para ahorrar dictadas técnicamente en las que Albert Hirschman pone énfasis en $(22,41 \mathrm{ss}$.).

14 Como Gerald Meier (31, p. 57) lo ha expresado: "El crecimiento de la población... simplemente desvía el capital a la manutención de niños que fallecen antes de alcanzar la edad productiva". Esta "formación de población" puede desde luego contribuir a la formación de capital humano que no está incluido en los datos de ahorro. Como se menciona después, sin embargo, también puede esperarse que cocientes de dependencia elevados reduzcan la inversión en educación, salud y adistramiento, que en la mayoría de los países subdesarrollados pueden ser áreas de formación de capital humano de alto beneficio.

15 La hipótesis no es necesariamente que las familias grandes ahorren menos que las familias pequeñas. En algunos países, el tamaño de la familia guarda relación directa con el ingreso, y las familias de altos ingresos pueden en verdad ahorrar mucho, relativamente. Es más, sus ahorros pueden ser aún mayores si se estandariza por tamaño de la familia. El estudio de Eizenga, referido más adelante, encontró este resultado en el ahorro personal en Estados Unidos.

1 M Modigliani (33, pp. 98-100) adoptó una línea de razonamiento similar con respecto a la sustituibilidad entre el ahorro de las empresas y el ahorro personal, y obtuvo evidencia estadística significativa en apoyo de esta hipótesis. Agradezco al profesor Modigliani el haberme facilitado su escrito mimeografiado. 
y de escala mínima grande. Estas grandes inversiones, que en otros países estarían financiadas por el ahorro de las empresas, pueden aparecer bajo el encabezado institucional de un excedente público o como préstamos del sector privado.

La hipótesis de una relación entre los cocientes de dependencia y las tasas de ahorro ha sido de hecho apoyada por estudios econométricos que incluyen factores demográficos. Un estudio de W. Eizenga (17) mostró que el tamaño de la familia y la distribución de edades de la población fueron factores estadísticamente significativos de explicación del ahorro personal en Estados Unidos. Normalizado para suprimir el efecto de estos factores, el cociente ahorro personal/ingreso fue superior al obtenido cuando esos factores no se incluían en el análisis. Un estudio más amplio, el análisis pionero de Modigliani sobre las tasas de ahorro "privado" en distintos países (ahorro privado más empresarial), también demostró la importancia de la distribución de Ia población por edades $(33,77 \text { ss. })^{17}$

El trabajo presente extiende el alcance de la investigación sobre las tasas de ahorro interno (por ejemplo, incluyendo el ahorro del sector público) y también considera un número de países mayor, 74, en comparación con 24 y 36 en la muestra de Modigliani. Aun más importante que la inclusión de más observaciones, el campo de variación es grandemente aumentado a medida que se incluyen más países desarrollados y subdesarrollados. Otro rasgo de este análisis, sugerido por razonamientos recientes, es la inclusión de varios países socialistas.

\section{ESTLMACIÓN ESTADÍSTICA}

La hipótesis delineada en la sección anterior puede ponerse en términos más formales de la manera siguiente. Si reescribimos la identidad del ingreso nacional con la igualdad de ahorro e inversión ex post e incluimos separadamente el consumo de la población en edad de trabajar, $C_{w a}, \mathrm{y}$ el gasto de la población en los dependientes $D$, tenemos

$$
Y=C_{w a}+D+S+X-M
$$

Al dividir por $Y$ y reacomodar términos se tendría que $S / Y$ estaría inversamente relacionada con la participación del gasto en dependientes en el ingreso nacional. Como se explica más adelante, las hipótesis pueden ser elaboradas teóricamente por varios caminos. Como primer paso, sin embargo, pareció valer la pena aclarar si ello tiene algún

17 Modigliani (33, 77 ss.) atacó el problema del crecimiento cle la población y el ahorro desde una posición algo distinta. En concordancia con la hipótesis del ciclo vital sobre el ahorro, planteó la hipótesis de que el ahorro (privado) debería ser función directa del crecimiento de la población. Esto se infiere a medida que el tamaño de cohortes de edad sucesivas que se incorporen a la población económicamente activa - que ahorran para cuando se retiren- creciera con el tiempo. Sin embargo, una vez hechas las pruebas estadísticas, él mismo afirma: "Es aparente que [los resultados] no dan ningún respaldo a la hipótesis... en verdad, su coeficiente es consistentemente negativo..." De esto, pasó a especificar pruebas basadas explícitamente en la carga de dependencia, y encontró resultados similares a los que se describen adelante. 
sentido empírico. Por lo tanto, el modelo estimado es algo simple, y busca probar la proposición de que la tasa del ahorro agregado de un país es menor, ceteris paribus, en la medida en que la población de éste tenga más dependientes.

La técnica adoptada fue el análisis de regresión múltiple. Las variables endógenas empleadas en las diferentes ecuaciones son $S / Y$, la tasa de ahorro interno agregado, y $S / N$, el nivel de ahorro per capita, expresado en dólares de 1964. Las variables exógenas empleadas son: primero, el nivel de ingreso per capita $Y / N$. Las observaciones en la mayoría de los países son para 1964, expresadas en dólares de Estados Unidos. Para algunos países hubo necesidad de emplear observaciones de otros años, pero expresadas también en dólares de Estados Unidos. Los detalles sobre fuentes y metodología acerca de todos los datos aparecen en el apéndice. Siguiendo la línea de trabajos recientes de Houthakker (24, p. 222), Modigliani (33, 55 ss.), Swamy (44) y Mizoguchi (32), también se introdujo la tasa de crecimiento del ingreso.18 La hipótesis original de Modigliani-Brumberg sugirió usar la tasa de crecimiento del ingreso agregado, $G_{1}$, pero como veremos adelante, $G_{2}$, la tasa de crecimiento del ingreso per capita, resultó ser más significativa. Ambas variables fueron representadas por la tasa media de crecimiento anual de los cinco años precedentes, expresada en puntos de porciento. 19

Se calculó $D_{1}$ como índice burdo de la carga de dependencia impuesta por los niños, el porciento de la población con edad menor o igual a 14 años, ${ }^{20}$ que muestra variación entre 40 y $46 \%$ en la mayoría de los países subdesarrollados, y 24 a $31 \%$ en los avanzados. También, de acuerdo con sugerencias teóricas, apoyadas por los resultados empíricos de Modigliani, acerca de que la población de más edad —los retirados- constituye asimismo una carga de dependencia por ser consumidores que no contribuyen corrientemente a la producción, fue introducida una variable adicional, $D_{2} .{ }^{21}$ Esta se definió como el por-

18 Los resultados de Swamy y de Mizoguchi se refieren al ahorro personal. 19 Claramente, puede existir el problema de decidir si la tasa de ahorros es influida por la tasa de crecimiento o si la causación va de la dependencia de la tasa de crecimiento sobre la tasa de inversión y por ende sobre el ahorro. Modigliani (33, pp. 68-75) trató con un problema algo similar (diferente en lo que se refiere al ahorro "privado" en vez del ahorro interno agregado), y de pruebas estadísticas concluyó que la relación entre ahorro y crecimiento puede ser identificada. En cualquier caso, como veremos más adelante, en la mayoría de las ecuaciones $G$ resulta no ser cuantitativamente muy importante como variable exógena.

20 El monto del gasto de dependencia dependerá del número de dependientes y del nivel de vida al que son mantenidos. La mayor parte del gasto en ellos, particularmente en los niños, es provisto por las familias. Como lo han señalado Duesenberry y Okun (15, pp. 233-234, 236), esta circunstancia limita el nivel de vida de dichos grupos, y lo relaciona con el nivel de consumo de la familia. En vista de la falta de información intermacional sobre el tema, supondremos como primera aproximación que la distribución de los dependientes por niveles de ingreso familiar es insesgada, y podemos entonces discutir su volumen de gasto en términos del número total de éstos en la población.

21 Ia inclusión de la población de mayor edad como factor reductor del ahorro puede justificarse sobre dos bases. Primera, debido a imperfección de sus previsiones, Jas personas de mayor edad pudieron no haber guardado lo suficiente para sus años no productivos (añádanse otras condiciones tales como la inflación y la imperfección del mercado de capital, los aumentos de la esperanza de vida y 
ciento de personas de 65 años o más. Dicha variable oscila entre 3 y $5 \%$ en la mayoría de los países subdesarrollados, y entre 8 y $13 \%$ en jos avanzados. Finalmente, se incluyó la tasa de dependencia total, $D_{3}$, que es la suma de $D_{1}$ y $D_{2}$. Se tuvo a la mano información demográfica adecuada de ahorros e ingreso sobre 74 paises: 47 subdesarrollados, 20 países occidentales desarrollados y 7 países socialistas de Europa oriental.

\section{Resultados de la regresión}

Primero se ajustaron, por minimos cuadrados ordinarios, ecuaciones logarítmicas para cada variable exógena considerada. Los resultados se presentan en el cuadro 2.

\section{Cuadro 2}

ESTIMACIÓN DE In $S / Y$ MEDIANTE REGRESIÓN SIMPLE PARA 74 PAÍSES

(Los números entre paréntesis son los errores estándar)

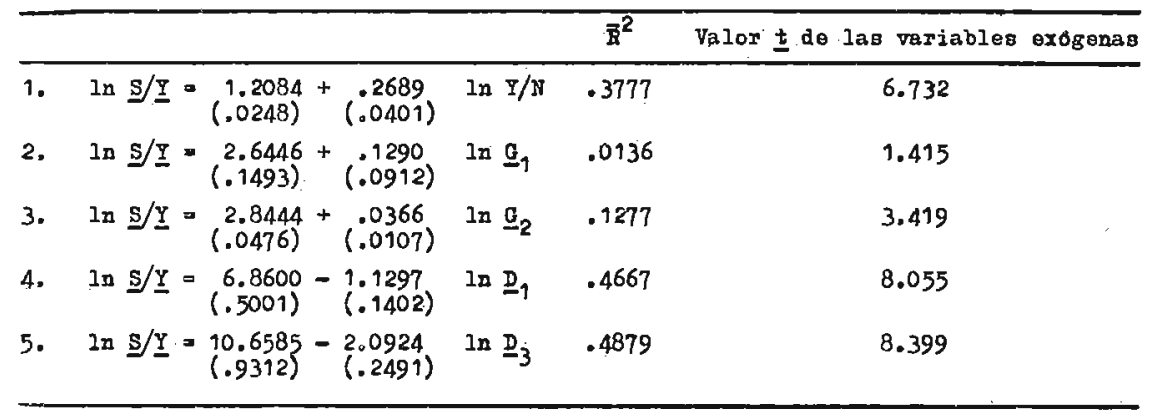

Como puede verse, los signos de los coeficientes de regresión son consistentes con lo esperado a priori y los valores $t$ son todos satisfactoriamente grandes. En lo que se refiere a las variables explicativas: $G_{2}$ no explica mucho de la varianza de $S / Y$, aunque sí más que $G_{1}$. El nivel de ingreso per capita se comportó mejor pero $D_{1}$ y $D_{3}$ redujeron el residuo aún más. ${ }^{22}$ La importancia de los factores de dependencia en la explicación de las tasas de ahorro aparece aun más claramente, sin embargo, en las regresiones múltiples.

$$
\begin{gathered}
\ln S / Y=\frac{7.3439+.1596 \ln Y / N+.0254 \ln G_{2}-}{(1.2819)(.0555)} \\
-\begin{array}{l}
1.3520 \ln D_{1}-.3990 \ln D_{2} \\
(.2913) \\
\vec{R}^{2}=.5697 \quad(.1586)
\end{array} \quad F=25.1604
\end{gathered}
$$

la elevación del nivel de vida "mínimo"), y por tanto constituyen una fuga de ingreso en el gasto discrecional de otros. Segunda, que pudieron haber estado gastando de su ahorro por lo cual su relación ahorro/ingreso corriente es muy baja. Por lo tanto, en cualquier punto del tiempo, ceteris paribus, una gran pro porción de retirados significaría un menor tasa de ahorro agregado.

22 En la misma forma fueron también estimadas ecuaciones lineales, y éstas 


$$
\begin{aligned}
& \ln S / Y=\frac{7.7762}{(1.3435)}+\underset{(.0449)}{.1037} \ln Y / N+\underset{(.0078)}{.0234 \ln G_{2}-} \\
& -1.4893 \ln D_{3} \\
& \text { (1.3071) } \\
& \bar{R}^{2}=.5729 \quad F=33.6357
\end{aligned}
$$

La hipótesis concerniente a los efectos de las condiciones de dependencia está sorprendentemente respaldada por los resultados de la regresión en el cuadro 3. Los pesos beta, que muestran la importancia relativa de las diferentes variables exógenas para explicar la varianza

\begin{tabular}{|c|c|c|c|}
\hline & $\begin{array}{c}\text { Elasticidad } \\
\text { de in } S / \underline{Y} \\
\text { con res } \\
\text { pecto } a\end{array}$ & $\begin{array}{c}\text { Valor } \frac{t}{d e} \text { regresion }\end{array}$ & Peso beta \\
\hline Ecuacion (1) & & (69 grados de libertad) & \\
\hline In $Y / \mathbb{N}$ & .1596 & 2.8776 & .3644 \\
\hline $\ln \quad \mathrm{g}_{2}$ & .0254 & $3: 2792$ & .2570 \\
\hline $\ln \quad \underline{D}_{1}$ & -1.3520 & 4.6406 & .7977 \\
\hline $\ln \quad \underline{D}_{2}$ & -.3990 & 2.5623 & .5107 \\
\hline Ecuacion (2) & & (70 grados de libértad) & \\
\hline $\ln \underline{Y} / \mathbb{N}$ & .1037 & 2.3132 & .2437 \\
\hline $\ln \quad \mathrm{G}_{2}$ & .0234 & 3.0428 & .2387 \\
\hline $\ln \underline{\mathrm{D}}_{3}$ & -1.4893 & 4.8493 & .5264 \\
\hline
\end{tabular}
de la variable endógena, indican que los términos $D$ contribuyen con

\section{Cuadro 3}

ESTADÍSTICAS DE REGRESIÓN DE $\ln S / Y$ PARA 74 PAİSES

la mayor parte. Los coeficientes de regresión, que en estas ecuaciones doblelogarítmicas indican elasticidades, son también mucho mayores para $\ln D_{1}$ y $\ln D_{3}$ que para los términos de ingreso, y sus valores $t$ son significativos por encima del nivel 0.005 .

No obstante, al interpretar estos resultados debemos darnos cuenta de la posible existencia de multicolinearidad. Las correlaciones simples entre $\ln Y / N$ y $\ln D_{1}, \ln D_{2}$ y $\ln D_{3}$, son respectivamente -.74, $.80 \mathrm{y}-.67$. Éstos no son tan bajos como es de desearse, pero como los valores $t$ lo indican, los coeficientes de regresión del ingreso per capita y las variables de dependencia fueron altamente significativos en todos los casos en que dichas variables se incluyeron juntas. Se puede ganar mayor seguridad del hecho de que los subconjuntos micas. 
de países considerados más adelante en este escrito dieron resultados similares, ya que con cobertura muestral diferente y con menor intercorrelación entre las variables exógenas, estas ecuaciones también mostraron elasticidades grandes y alto nivel de significación estadística para las variables de dependencia. ${ }^{23}$

También se ajustaron ecuaciones en las que el nivel de ahorro per capita fue tomado como variable endógena.

$$
\begin{aligned}
& \ln S / N=\underset{(1.2340)}{2.7851}+\underset{(.0534)}{1.1486} \ln Y / N+\underset{(.0075)}{.0265} \ln G_{2}- \\
& -1.3438 \ln D_{1}-.3966 \ln D_{2} \\
& \text { (.2805) (.1498) } \\
& \vec{R}^{2}=.9559 \quad F=396.677
\end{aligned}
$$

$$
\begin{aligned}
& \ln S / N=\underset{(1.2911)}{3.2383}+\frac{1.0924 \ln Y / N}{(.0431)}+\underset{(.0074)}{.0245} \ln G_{2}- \\
& -1.4857 \ln D_{3} \\
& \text { (.2953) } \\
& \bar{R}^{2}=.9563 \quad F=533.913
\end{aligned}
$$

Igual que en otros estudios de sección transversal del ahorro per aapita, $\bar{R}^{2}$ es relativamente alto. De mayor pertinencia en el presente contexto es que estos resultados demuestran claramente la importancia de las variables de dependencia como influencia cuantitativa muy importante y estadísticamente distinta sobre el ahorro. Los valores $t$ para los términos $D$ y para los de ingreso en las ecuaciones (3) y (4) son significativos por encima del 0.005. Más aún, los coeficientes de regresión relativamente grandes de $D_{1}$ y $D_{3}$ indican elasticidades del ahorro mayores respecto a los términos de dependencia que a los de ingreso per capita. Los coeficientes de regresión relativamente grandes de los términos $D$, en comparación con los $Y / N$, también apoyan la sugerencia de que estudios sobre niveles de ahorro que muestran la abrumadora importancia del ingreso como variable explicativa están en parte recogiendo el efecto'de condiciones demográficas no especificadas.

Otro resultado interesante es el valor del coeficiente de regresión en el término per capita, que indica una elasticidad-ingreso del ahorro cercana a la unidad. Este hallazgo apoya el trabajo de Modigliani (3) y de Friedman (21) y está también en estrecha concordancia con la

28 Los resultados de las ecuaciones (1) y (2) también indicaron por qué $Y / N$ no es una variable sustituto perfecta para la carga de dependencia a pesar de la alta correlación entre el ingreso per capita y la tasa de natalidad y entre ésta y $D_{1}$. A niveles de ingreso bajos, $D_{1}$ es elevada, pero $D_{\mathfrak{g}}$ es baja. Aunque $D_{1}$ es mucho más baja a niveles altos de ingreso per capita, $D_{2}$ es más elevada. En vista de que la carga total de dependencia, $D_{3}$, es el resultado neto de los dos efectos, no varía con el ingreso per capita tanto como lo hacen cualquiera de las otras dos cargas de dependencia por separado. 
ausencia de una tendencia ascendente en las relaciones $S / Y$ que hicimos notar en la introducción.

\section{DISCUSIÓN ADICIONAL}

Teniendo en cuenta la metodología y lo crudo de los datos, ${ }^{24}$ estos resultados pueden verse como apoyo de la noción de que las tasas de fecundidad y los factores de dependencia son determinante importante de las tasas de ahorro agregado.

Debemos notar también que el modelo que hernos empleado no hace completa justicia a las hipótesis que se intenta probar. Las variables exógenas se relacionan con la oferta de ahorro. Debido a las limitaciones impuestas por los datos disponibles, sin embargo, las variables endógenas se refieren a ahorro e inversión ex post. No se construye implícitamente en el modelo ninguna demanda de ahorro (aunque la variable de crecimiento, $G_{2}$, puede tal vez recoger condiciones de demanda tales como la presión sobre la capacidad productiva). Esta limitación es especialmente grave ya que la hipótesis basada en el peso de la carga de dependencia en el gasto discrecional se relaciona con el potencial del ahorro. Seguramente los ahorros reales pudieron haber sido mayores en muchos países con condiciones de demanda más favorables. Nuestro enfoque de una ecuación, no obstante, como la mayoría de otros estudios estadísticos sobre el comportamiento de los ahorros entre los países, omiten lo anterior.

Al interpretar nuestros resultados, debemos darnos cuenta de otro problema posible. No está enteramente claro si la relación sobre la que nos hemos dirigido vincula el comportamiento del ahorro y las condiciones demográficas, o si no hemos relacionado meramente dos de los muchos factores que constituyen el complejo del subdesarrollo. Bajo esta perspectiva, cualquier otra condición de éstas - por ejemplo, bajo consumo de proteínas- podría comportarse igualmente bien en la "explicación" estadística de las tasas.25

Para considerar esta posibilidad, la ecuación (1) fue reestimada con una variable dummy que tomó valor 1 para países subdesarrollados y cero pará los desarrollados.

24 Acerca de las deficiencias de los datos sobre inversión y ahorro en los pá́ses subdesarrollados véase, por ejemplo, Richard Hooley (10). Del mismo modo, mejores resultados se obtendrían probablemente si se definiera $D_{1}$ en forma distinta para los países desarrollados y los subdesarrollados. En el caso de los primeros una medida más realista de la carga de dependencia infantil podría ser el porciento de la población de menos de 18 años y, en algunos países, hasta menor de 21 . Aun cuando esta población adolescente se mantiene en parte por sí misma, el nivel absoluto registrado de sus gastos de consumo (incluyendo educación secundaria y superior) es mucho más alto que para los niños, y el impacto sobre el gasto discrecional y el ahorro registrado es correspondientemente superior.

25 La misma objeción y con igual validez puede hacerse a los modelos econométricos que usan el ingreso per capita como variable explicativa, ya que en ese plan el ingreso bajo es simplemente uno de los muchos rasgos interrelacionados del subdesarrollo. Como el ejemplo del ingreso lo indica, sin embargo, hay una diferencia importante si la variable empleada se ha seleccionado también sobre bases analíticas, lo cual hace posible una vinculación teórica entre las variables. Como se estableció en la sección II, este criterio se satisface en el caso de las tasas de dependencia y ahorro. 


$$
\begin{aligned}
& \ln S / Y=\underset{(1.2214)(.1241)}{6.6757}-.3980 D U M M Y+\underset{(.0568)}{.8272} \ln Y / N+ \\
& +.0228 \ln G_{2}-.9713 \ln D_{1}-.3944 \ln D_{2} \\
& \begin{array}{lll}
(.0073) & (.2982) & (.1462)
\end{array} \\
& \bar{R}^{2}=.6207 \quad F=24.8933
\end{aligned}
$$

\begin{tabular}{llc}
\hline Variable & Valort & Peso beta \\
\hline & 5.4657 & - \\
$D U M M Y$ & 3.2068 & - \\
$\ln Y / N$ & 1.5362 & .205 \\
$\ln G_{2}$ & 3.1139 & .233 \\
$\ln D_{1}$ & 3.2576 & .573 \\
$\ln D_{2}$ & 2.6978 & .505 \\
\hline
\end{tabular}

El valor $t$ de la variable dummy es significativo por encima del nivel 0.005 . Esto indica, lo cual no debe extrañar, que otros aspectos del subdesarrollo, aparte de las condiciones de dependencia, influyen sobre las tasas de ahorro agregado. Pero los coeficientes en $\ln D_{1}$ y $\ln D_{2}$ retienen también su significación estadística al nivel 0.005 . Y mientras la inclusión de la variable dummy reduce la magnitud del coeficiente de regresión de $\ln D_{2}$ (y de $\ln Y / N$ ) las elasticidades de las variables de dependencia continúan siendo muy altas.

El nivel de significación de la variable dummy en la ecuación (5), sin embargo, sugiere que puede haber diferencias importantes en el comportamiento del ahorro entre los países desarrollados y los subdesarrollados. Por tanto, fueron ajustadas ecuaciones separadas a los 47 países subdesarrollados y a los 20 países occidentales avanzados. ${ }^{26}$ Las estimaciones de los parámetros aparecen en el cuadro 4.

Como puede verse, en ambos grupos de países las variables de dependencia son significativas estadísticamente, y sus coeficientes de regresión indican que son cuantitativamente más importantes que los términos ingreso en la determinación de las tasas de ahorro.agregado. ${ }^{27}$ En las ecuaciones del nivel de ahorro per capita, los coeficien-

26 Se presentó el problema de si incluir los 7 países socialistas en el subconjunto de países occidentales desarrollados. La aplicación de la prueba de Chow para la covarianza (6) llevó al rechazo (tenue) de la hipótesis nula, esto es, que estas observaciones pertenecen a una estructura distinta de regresión. Pero como se tenían muy pocos grados de libertad que permitieran tener confianza sustancial en la prueba y debido a dudas concernientes a la comparabilidad de los datos, no incluí esos países en el subconjunto mencionado.

27 A propósito del posible problema de multicolinearidad mencionado antes, el mismo patrón de coeficientes de regresión que apareció en las primeras ecuaciones reaparece aquí no obstante la cobertura muestral diferente y la menor correlación entre el ingreso per capita y las variables de dependencia. Para los 47 paises subdesarrollados, la correlación simple entre $\ln Y / N$ y $\ln D_{1}$ y $\ln D_{3}$ es -.4211 y .5174 , respectivamente. Para los 20 países desarrollados, los coeficientes correspondientes son .1121 y -.0324 . La posible multicolinearidad que hemos notado en nuestras ecuaciones señala una advertencia concerniente a los estudios de sección transversal sobre ahorro que incluyen un término de ingreso, pero 


\section{Cuadro 4}

ESTIMACIONES DE PARAMETROS DE AHORRO EN 47 PAISES SUBDESARROLLADOS Y 20 PAISES OCCIDENTALES DESARROLLADOS

(Los errores estándar están entre paréntesis)

\begin{tabular}{|c|c|c|c|c|c|c|}
\hline \multirow{2}{*}{$\begin{array}{l}\text { Variables } \\
\text { endogenas }\end{array}$} & \multicolumn{4}{|c|}{ Variables exogenas } & \multirow{2}{*}{$\underline{\bar{R}}^{2}$} & \multirow[b]{2}{*}{$\underline{P}$} \\
\hline & $\ln . Y / N$ & $\ln \mathrm{g}_{2}$ & $\ln \underline{D}_{1}$ & $\ln \underline{D}_{2}$ & & \\
\hline \multicolumn{7}{|l|}{$\begin{array}{l}\text { Palsor sub- } \\
\text { desarrolla- } \\
\text { dos }\end{array}$} \\
\hline 1. In $\underline{S} / \underline{Y}$ & $\begin{array}{l}.1292^{t} \\
(.0669)\end{array}$ & $\left(.0227^{\text {tht }}\right.$ & $\begin{array}{c}-1.2297^{t} \\
(.4703)\end{array}$ & $\begin{array}{l}-.4455^{\text {th }} \\
(.2067)\end{array}$ & .2419 & 4.6685 \\
\hline 2. In $\underline{S} / \mathbb{N}$ & $\begin{array}{l}1.1167^{\text {dx }} \\
(: 6634)\end{array}$ & $\left(\begin{array}{l}.0239 \\
.0077)\end{array}\right.$ & $\begin{array}{c}-1.3122^{ \pm 1} \\
(.4463)\end{array}$ & $\begin{array}{l}-.4469^{t t} \\
(.1962)\end{array}$ & .8975 & 101.6727 \\
\hline \multicolumn{7}{|l|}{$\begin{array}{l}\text { Palaen Occi } \\
\text { dentales des } \\
\text { arrollados }\end{array}$} \\
\hline 3. $\ln \underline{S} / \underline{\underline{Y}}$ & $\begin{array}{l}.0035 \\
(.1198)\end{array}$ & $\begin{array}{l}.2589^{\star} \\
(.1595)\end{array}$ & $\begin{array}{c}-.4324^{k t} \\
(.2529)\end{array}$ & $\begin{array}{l}-.4916^{t t} \\
(.1852)\end{array}$ & .4395 & 4.7245 \\
\hline 4. $\ln \underline{S} / \mathbb{N}$ & $\begin{array}{l}1.0049^{\star t k} \\
(.1201)\end{array}$ & $\begin{array}{l}.2591^{\hbar} \\
(.01599)\end{array}$ & $-. .4300)$ & $\begin{array}{l}-.4914 \\
(.1856)\end{array}$ & .8413 & 26.1798 \\
\hline
\end{tabular}

* Significativo por encima del nivel .1.

$*$ Significativo por encima del nivel 0.01 .

tes de regresión del ingreso per capita continúan indicando elasticidadingreso cercana a la unidad -ligeramente más en los países subdesarrollados y casi exactamente 1 en los desarrollados. Así, los resultados de subconjuntos confirman el patrón general de comportamiento del ahorro observado en toda la muestra. ${ }^{.8}$

\section{IMPLICACIONES ANALÍTICAS}

Estos hallazgos que muestran la importancia de las condiciones de dependencia como determinantes de las tasas de ahorro agregado pueden ser conceptualizados de diferentes maneras. Primero, podríamos plantear un modelo en el que los individuos maximizan una utilidad que es función del consumo presente y futuro, y de los niños. Sin embargo, considerando la limitada disponibilidad de dispositivos y

que no especifican variables de dependencia. Como es bien sabido (véase Farrar y Glauber, 19, p. 106), los problemas de multicolinearidad en la regresión pueden surgir no sólo cuando las variables colineales están incluidas sino también cuando no lo están. Así, los estudios de sección transversal sobre ahorro que no incluyen explícitamente variables de dependencia pueden dar lugar a estimaciones sesgadas de los parámetros, debido a la falta de especificación.

28 La diferencia más importante con los resultados de todo el conjunto de países es la elevada elasticidad de $\ln S / Y$ respecto de $\ln G_{2}$ en el caso de los páses desarrollados. 
conocimientos contraceptivos en la mayoría de los países subdesarrollados y algunos desarrollados durante este periodo, esto podría no ser realista porque las rigideces tecnológicas (fisiología humana) pueden haber interferido el comportamiento "optimizante". ${ }^{29}$ Un enfoque alternativo sería simplemente integrar nuestros hallazgos con teorías corrientes sobre el ahorro, sujetas a las restricciones impuestas por el gasto en los dependientes. La elaboración teórica de tal modelo, podría, a su vez, proceder por otros caminos..$^{30}$

Nuestros resultados pueden ser útiles no sólo para comprender el comportamiento reciente del ahorro en los países desarrollados y los subdesarrollados, sino en otros contextos igualmente. Los resultados de regresión pueden ayudar a tomar en cuenta la ausencia de aumentos, desde 1870 , de las tasas de ahorro agregado en la mayoría de los países occidentales avanzados. ${ }^{31}$ Aunque el ingreso per capita en estos países se ha triplicado o cuadruplicado (30, pp. 201-206), sus tasas de dependencia totales han sido relativamente constantes (ibid., p. 31). La evidencia geográfica es también consistente con la hipótesis de que las condiciones demográficas favorables pueden haber sido uno de los factores importantes que hicieron posibles las relativamente elevadas tasas de ahorro de los países socialistas de Europa oriental. ${ }^{32}$ Como las tasas de fecundidad en esos países (con excepción de la URSS) han alcanzado niveles que son bajos en comparación con las de la mayoría de los países subdesarrollados antes de que los comunistas tomaran el poder, esto sugiere que los rasgos institucionales especiales de estos

29 Los interesados en el análisis económico del comportamiento de la fecundidad bajo supuestos de elección pueden consultar a Gary Becker (4). Desde luego, no está muy claro si las tasas de fecundidad elevadas se deben a falta de conocimiento y de acceso a técnicas contraceptivas modernas. El estudio de Paul Schultz sobre las tasas de fecundidad en Puerto Rico (39) da el mismo respaldo a la hipótesis de que en las sociedades rurales y pre-modernas un mayor tamaño de la familia puede ser respuesta racional a los beneficios y costos económicos de la paternidad en tales medios.

30 Por ejemplo, los individuos pueden reaccionar ante la perspectiva de gastar en los niños haciendo ahorros anticipados, para así reducir la carga corriente cuando se presente. Mejorar las especificaciones de la restricción impuesta por el gasto de dependencia requeriría probablemente mejor información sobre las cuestiones que fueron consideradas en forma preliminar en la nota 20 . De manera semejante, la influencia de la carga de dependencia sobre las formas de ahorro distintas al ahorro familiar requerirían mayor conocimiento de las posibilidades de sustitución y de los efectos del ahorro en el intercambio a través de las diferentes formas institucionales.

31 Véase Kuznets (26, pp. 235-240). Después de 1950, con la aceleración de las tasas de crecimiento de estos países, las tasas de ahorro agregado sí se elevaron. Esto es consistente con nuestros resultados, aunque la dirección de causalidad no es muy clara.

32 Digo "uno" porque en la ecuación (2), en el caso de los países socialistas, los residuos no resultaron distribuidos aleatoriamente. Las observaciones reales de 6 de los 7 países socialistas incluidos en la muestra quedaron por encima de la superficie de regresión, y los residuos promediaron $24 \%$. También se estimó para los países socialistas una ecuación separada, y se hizo la prueba propuesta por Chow (6) para ver si estos países pertenecían, en el sentido estadístico, al mismo universo que generó la pendiente de la ecuación calculada con todos los países. La prieba no fue concluyente. A un nivel de significación del $5 \%$, fue posible rechazar la hipótesis de que estas observaciones eran parte del mismo modelo de regresión. No fue así al nivel de $1 \%$. 
regímenes pueden no ser condición suficiente para alcanzar tasas de ahorro de esa magnitud.33

\section{IMPLICACIONES DE POLÍTICA}

Este trabajo sugiere que las elevadas tasas de crecimiento de la población constituyen una barrera doble al desarrollo económico. Por una parte, como ha sido señalado a menudo, una población creciente aumenta el volumen de gastos de desarrollo necesarios para mejorar la calidad de la mano de obra y para elevar las tasas de capital por unidad de fuerza de trabajo.34 Nuestros resultados indican que a través de sus efectos sobre las tasas de dependencia, las tasas de fecundidad elevadas limitan también la capacidad de una economía para responder a esas necesidades adicionales de ahorro e' inversión. ${ }^{35}$

Nuestros resultados se relacionan sólo con el efecto de la carga de dependencia sobre la formación de capital físico, pero estas condiciones demográficas también afectan la formación de capital humano. Cuando menos, un gran número de niños dependientes introduce un sesgo en contra de la inversión en capital físico, porque independientemente de las tasas de beneficio, la moralidad convencional dicta usualmente que deben hacerse esfuerzos para mantener vivos a los niños a un nivel mínimo. Este gasto puede ser bajo por cada niño, pero multiplicado por un gran número de niños puede absorber una gran proporción de los recursos potencialmente disponibles para el ahorro y la inversión. Asimismo, a causa de la prioridad dada usualmente a la sobrevivencia física de los niños, puede no haber recursos suficientes para mejorar la calidad del factor humano mediante inversiones en educación y adiestramiento. Como resultado, una carga de dependencia elevada puede llevar a una abundancia económicamente subóptima de fuerza de trabajo "cruda" en relación con la fuer-

33 Esto sugiere que si los regímenes socialistas hubieran tomado el poder en países sin estas condiciones demográficas favorables, tal vez no hubieran podido alcanzar tasas de ahorro e inversión tan heroicas. El coeficiente de inversión bruta de Cuba, del periodo 1961-1963, ha sido estimado aproximadamente en $18 \%(77,0.218)$. No está muy claro si éste es el cociente referido al producto bruto nacional o al producto material neto. Si se refiriera a este último, ello implicaría un coeficiente inversión/ingreso nacional menor; y como el flujo de capital extranjero fue positivo, una tasa de ahorro agregado aun menor. En cualquier caso, estas cifras son difícilmente comparables con las tasas alcanzadas por los países socialistas de Europa oriental. Y el cociente de dependencia cubano, $D_{3}$, fue bajo en comparación con el de la mayoría de los países subdesarrollados.

34 En el ejemplo típico: con población que crece al $3 \%$ anual y relación capital/producto igual a 4 , un país debe ahorrar el $12 \%$ del producto nacional bruto sólo para mantener constante el nivel existente de ingreso per capita. Si se supone una tasa de ahorro agregado relativamente alta de $18 \%$, tomaría 35 años duplicar dicho ingreso.

85 Nuestros hallazgos agudizan la observación frectiente de que las altas tasas de fecundidad en los países subdesarrollados constituyen una desventaja para el desarrollo, desventaja a la que la mayoría de los paises avanzados de la actualidad no tuvieron que enfrentarse durante su transición al desarrollo económico. El promedio de tasas de fecundidad de los 47 países subdesarrollados considerados aquí fue aproximadamente $25 \%$ más alto que el correspondiente a un grupo de 10 países occidentales europeos a mediados del siglo xax (7, pp. 68-69). 
za de trabajo calificada, y con el acervo de capital físico. Tal situación se observa ya en muchos países subdesarrollados.

Nuestra discusión sugiere, entonces, que las tasas elevadas de fecundidad y las cargas también elevadas de dependencia, en las condiciones de la mayoría de los países subdesarrollados, pueden implicar una asignación subóptima de recursos debido a rigideces fisiológicas y/o institucionales. Esto es, los niños nacen de padres que podrían preferir no haberlos tenido, pero que están de allí en adelante destinados a mantenerlos. Estos dependientes absorben una gran proporción de los recursos potencialmente disponibles para inversión física y para las áreas de formación de capital humano de alto beneficio.

Las implicaciones de estas condiciones demográficas son aún más inquietantes porque las tendencias presentes en muchos países subdesarrollados parecen estar llevando a tasas de dependencia altas más bien que bajas. En 1953, las tasas de dependencia de equilibrio (esto es, la distribución por edades que resultaría a la postre, dadas las tasas de fecundidad y mortalidad existentes e independientemente de las condiciones iniciales) de las poblaciones de Latinoamérica, Asia y Africa eran aproximadamente $10 \%$ más altas que las tasas actuales (1, p. 334). Más aún, las tasas de dependencia de equilibrio han estado elevándose, porque con mayores niveles de ingreso, en los últimos decenios las tasas de fecundidad en la mayoría de los países subdesarrollados han aumentado en lugar de disminuir (12, pp. 27-28) (45). E1 problema se agrava porque cuando en los países subdesarrollados las tasas de mortalidad caen, las más beneficiadas no son las cohortes de mayor edad, sino las más jóvenes. Esta reducción de la mortalidad de niños y de la infantil se agrega a la carga de dependencia y constriñe aún más el potencial de ahorro de la economía.

Si estas conclusiones son interpretadas con miras a apoyar los esfuerzos de política dirigidos a reducir las tasas de fecundidad en los países subdesarrollados, deben ser distinguidos dos casos muy diferentes. Primero, en algunas áreas, y notablemente ciudades en sociedades en transición, la motivación para tener menos nacimientos está presente -como lo evidencian las altas tasas de aborto inducido. En estos casos, los programas para hacer disponibles técnicas más fáciles de reducción de nacimientos deberían ser suficientes, aunque la escala y alcance del esfuerzo requerido pueden tener que ser mayores que los contemplados en los programas corrientes de planeación de la población. ${ }^{36}$ En otras áreas, sin embargo, el problema puede estar en crear las condiciones para estimular nuevas actitudes hacia la elección del tamaño de la familia.ji Además, deberíamos apuntar

30 Adviértase el pesimismo con que Kingsley Davis se ha expresado en relaciớn a los programas de población presentes (13).

37 La investígación de Irma Adelman sobre los determinantes de las tasas de fecundidad internacionales indica direcciones semejantes de la política. La señora Adelman probó un modelo en el que la tasa de fecundidad a una edad específica fue la variable endógena, y el ingreso per capita, la urbanización, la educación (en gran parte el alfabetismo), y la densidad de población las variables exógenas. La elasticidad de las tasas de fecundidad por edad respecto a la educación fue mayor que con respecto a las otras variables, y más significativa para los países subdesarrollados que para los desarrollados (1, pp. 321-323, 336-337). Para facilitar el diseño de proyectos en esta área se necesitan ulteriores investigaciones que 
que en esta situación podría considerarse un enfoque en que el control de la población dependiera menos de la voluntad. Aun en casos en que los beneficios privados pudieran justificar familias de gran tamaño (39), las altas tasas de dependencia muestran un ejemplo clásico de una deseconomía tecnológica (no de mercado) externa: en países en que el ahorro es la restricción dominante sobre el crecimiento, la actividad procreativa de los individuos reduce el bienestar social al disminuir la tasa de ahorro agregado y la tasa de desarrollo. Por lo tanto, una política activamente intervencionista con incentivos y "desincentivos" para reducir las tasas de fecundidad quedaría justificada en criterios generales de bienestar.

\section{CONCLUSIONES}

Este trabajo provee apoyo estadístico a la sugerencia adelantada por un número de economistas de que las condiciones demográficas son un determinante "principal" de las tasas de ahorro agregado. Nuestros resultados indican que los cocientes de dependencia son una influencia estadísticamente distinta y cuantitativamente importante sobre las tasas de ahorro agregado. Esto es cierto para el total de 74 países considerados y para los subconjuntos de subdesarrollados y desarrollados. La especificación de las variables de dependencia redujo considerablemente la varianza no explicada de $S / Y$ aunque es obvio que queda mucho trabajo por hacer en este terreno. ${ }^{38}$

Las altas tasas de dependencia - y en última instancia las tasas de natalidad- están entre los factores importantes que explican la gran disparidad entre las tasas de ahorro agregado de los países desarrollados y los subdesarrollados. El ingreso per capita no lo hace. El análisis de sección transversal mostró elastiçidad-ingreso del ahorro cercana a la unidad. Estos resultados, y la estabilidad (si no tendencia ascendente) de las tasas de natalidad y de dependencia, ayudan a explicar la ausencia de crecimiento de las tasas de ahorro agregado en función de niveles crecientes de ingreso en la mayoría de los países subdesarrollados. ${ }^{39}$

Nuestro análisis indica pesimismo con relación a la posibilidad de que los países subdesarrollados alcancen aumentos sustanciales en sus tasas de ahorro a menos que las tasas de fecundidad disminuyan. Sobre lo último, como es de notar, no hay bases para sentirse optimistas. Particularmente en algunos países de menor desarrollo, los

provean información más detallada sobre los efectos de la educación en el cormportamiento de la fecundidad. Mientras tanto, las conclusiones de Irma Adelman respaldan las sugerencias que se han hecho sobre otras bases, sobre la mayor prioridad que debe darse a la política de programas de alfabetización rural. Sin embargo, como señaló un lector de una versión anterior de este escrito, dar énfasis a la educación para que la gente pueda leer acerca de la píldora es un rodeo muy largo cuando se puede dar mayor beligerancia, en las discusiones actuales, a un enfoque directamente intervencionista como se hace notar más adelante.

38 Aún dentro del contexto de modelos de una sola ecuación la información disponible en el presente no permite mayor especificación de variables tan relevantes como las tasas de beneficio, la eficiencia del mercado de capital, la distribución del ingreso por niveles y la preferencia-tiempo.

39 Esta cuestión se trata de manera muy diferente pero complementaria en (29). 
programas públicos de control de natalidad han sido a menudo obstaculizados por opiniones nacionalistas de los intelectuales y de la élite, incluyendo muchos economistas. Los resultados presentes pueden ser pertinentes para convencer a estas personas de que al limitar su capacidad para la formación de capital fijo - una meta que es ampliamente deseada- el crecimiento rápido de la población puede estar en conflicto con sus propias metas de desarrollo.

\section{APÉNDICE}

\section{Fuentes de datos y metodología}

Se tuvieron disponibles los datos demográficos y de ingreso nacional de los siguientes 74 países: Costa Rica, El Salvador, Puerto Rico, Honduras, Nicaragua, República Dominicana, Jamaica, Trinidad y Tobago, Panamá, Argentina, Brasil, Chile, Ecuador, México, Paraguay, Perú, Venezuela, Uruguay, Malta, Chipre, Grecia, España, Portugal, Turquía, Irán, Barbados, Guayana Inglesa, Egipto, Túnez, Uganda, Mauritania, Tangañika, Ghana, Sudán, Kenya, Irak, Jordania, Israel, Marruecos, Ceilán, India, Pakistán, Tailandia, China (Taiwan), Corea del Sur, Malasia, Filipinas, Japón, Bélgica, Luxemburgo, Francia, Alemania Occidental, Italia, Holanda, Dinamarca, Islandia, Noruega, Suecia, Finlandia, Austria, Irlanda, Gran Bretaña, Suiza, Australia, Nueva Zelandia, Canadá, Estados Unidos, URSS, Hungría, Bulgaria, Checoslovaquia, Yugoslavia, Alemania Oriental y Polonia.

Los datos sobre población y cocientes de dependencia fueron calculados del Demographic Yearbook, 1965 de las Naciones Unidas (45). La información de 1964 sobre producto bruto y ahorro interno de aproximadamente 40 países subdesarrollados fue obtenida de la División de Estadística y Reportes de la Oficina de Coordinación de Programas de la Agencia para el Desarrollo Internacional, de Estados Unidos. El ahorro interno bruto fue medido como la diferencia entre la inversión bruta y el saldo de la balanza de pagos en cuenta corriente. En los casos en que las transferencias internacionales fueron elevadas en relación con la inversión bruta - por ejemplo, Jordania y Bolivia - estos datos fueron ajustados por las oficinas respectivas de la AID, de acuerdo con la versión revisada de la metodología de la contabilidad del ingreso nacional de las Naciones Unidas en su aspecto referente a la imputación de las transferencias internacio nales para usos de inversión o de cualquier otra índole. Las cifras en moneda local fueron convertidas a los tipos de cambio oficiales, excepto en los casos en que las oficinas de la AID consideraron más apropiadas otras tasas con base en criterios de paridad de poder de compra. Respecto a los países no-socialistas restantes, la información sobre ahorro y producto interno bruto fue tomada del Yearbook of National Income Accounts Statistics de las Naciones Unidas (49).

Para los países desarrollados se empleó la información del cuadro $9 \mathrm{~A}$ que contiene las monedas locales convertidas según los tipos oficiales de cambio. Para los países subdesarrollados se usaron los coeficientes de conversión del cuadro 9B, obtenidos a partir de tasas de paridad de poder de compra. La información sobre las tasas de tendencia de crecimiento del ingreso nacional se tomó de (49) y (50).

Los datos sobre producto bruto y ahorro interno en los países socialistas son de 1955, pero están expresados en dólares de 1964. Las estimaciones del producto bruto interno al costo de los factores y de su crecimiento fueron tomadas de Stanley Cohen (11); Maurice Ernst (18); y Frederick Pryor y George Staller (38). Las cifras de Polonia, Hungria, Checoslovaquia y 
la URSS sobre participación de la inversión bruta en el producto bruto interno al costo de los factores son de Thad Alton (3, p. 92); de Bulgaria de (18); de Alemania Oriental, de Wolfgang Stolper (42, p. 437); y de Yugoslavia, a precios de mercado, del cuadro A-35 en (46).

\section{REFERENCIAS BIBLIOGRAFICAS}

1. Irma Adelman, "An Econometric Analysis of Population Growth", American Economic Review, junio de 1963, 53, pp. 314339.

2. - y Cynthia Taft Morris, "A Quantitative Study of Social and Political Determinants of Fertility", Economic Development and Cultural Change, enero de 1966, pp, 129-157.

3. Thad Paul Alton, et al., Polish National Income and Product in 1954, 1955 and 1956, Nueva York, 1965.

4. Gary Becker, "Economic Analysis of Fertility", en National Bureau of Economic Research, Demographic and Economic Change in Developed Countries, Princeton, 1960.

5. Hollis B. Chenery y Peter Eckstein, "Development Alternatives for Latin America", Journal of Political Economy, de próxima publicación.

6. Gregory C. Chow, "Tests of Equality Between Sets of Coefficients in Two Iinear Regressions", Econometrica, julio de 1960, 28, 591-605.

7. Cambridge Economic History of Europe, VI. The Industrial Revolutions and After, Parte I, Cambridge, 1965.

8. A. J. Coale, "How the Age Distribution of a Human Population is Determined", Cold Springs Harbor Symposia on Quantitative Biology, 1967.

9. - "The Effects of Changes in Mortality and Fertility on Age Composition", Milbank Memorial Fund Quarterly, enero de 1956, 34.

10. - y E. Hoover, Population Growth and Economic Development in Low Income Countries, Princeton, 1958. (Crecimiento de la población y desarrollo económico, México, Limusa-Wiley, 1965.)

11. Stanley H. Cohen, "Soviet Growth and Retardation", en U. S. Congress, Joint Economic Committee, New Directions in the Soviet Economy, Washington, 1966.

12. O. A. Collver, Birth Rates in Latin America, Berkeley, 1965.

13. Kingsley Davis, "Population Policy: Will Current Programs Succeed?", Science, Núm. 3802, noviembre 10, 1967, pp. 730-740.

14. Paul Demeny, "Early Fertility Decline in Austria-Hungary: A Lesson in Demographic Transition", Daedalus, primavera de 1968 , pp. 502-522.

15. Demographic Change in Developed Countries, National Bureau of Economic Research, Princeton, 1961.

16. James S. Duesenberry, Income, Saving, and The Theory of Consumer Behavior, Cambridge, 1949.

17. W. Eizenga, Demographic Factors and Savings, Amsterdam, 1961.

18. Maurice Ernst, "Postwar Economic Growth in Eastern Europe", en U. S. Congress, Joint Economic Committee, New Directions in the Soviet Economy, Washington, 1966.

19. Donald E. Farrer y Robert R. Glauber, "Multicollinearity in Regression Analysis: The Problem Revisited", Review of Economic Statistics, febrero de 1967, pp. $92-108$.

20. Ronald Freedman, "The Transition from High to Low Fertility: Challenge to Demographers", Population Index, octubre de 1965, 31, pp. 417-430.

21. Milton Friedman, A Theory of the Consumption Function, Princeton, 1957.

22. Albert Hirschman, The Strategy of Economic Development, New Haven, 1958.

23. Richard Hooley, "The Measurement of Capital Formation in Underdeveloped Countries", Review of Economic Statistics, mayo de 1967, 49, pp. 199-208.

24. H. S. Houthakker, "On Some Determinants of Savings in the Developed and Underdeveloped Countries", en E. A. G. Robinson (compilador), Problems of Economic Development, 'Londres, 1965.

25. Charles P. Kindleberger, Economic Development, 2: ed., Nueva York, 1965.

26. Simon Kuznets, Modern Economic Growth, New Haven, 1966. 
27. - "Quantitative Aspects of the Economic Growth of Nations: Capital Formation Proportions", Economic Development and Cultural Change, 8, 4, II, 1960.

28. Luis Landau, "Determinants of Savings in Latin America" (Project for Quantitative Research on Economic Development, Harvard University, 1966), pp. 1-26.

29. Nathaniel H. Leff, "Marginal Savings Rates in the Development Process: The Brazilian Experience", Economic Journat, de próxima publicación.

30. Angus Maddison, Economic Growth in the West, Nueva York, 1964.

31. Gerald Meier, "The Problem of Limited Economic Development", Economia Internazionale, 1953, reimpreso en A. N. Agarwala y S. P. Singh (compiladores), The Economics of Underdevetopment, Nueva York, 1963.

32. T. Mizoguchi, "International Comparison of Savings Ratios by Occupational Groups", I, Hitotsubashi Journal of Economics, febrero de 1967, pp. 61-74.

33. Franco Modigliani, "The Life Cycle Hypothesis of Saving, the Demand for Wealth, and the Supply of Capital" (trabajo presentado al Congreso de la Sociedad Econométrica, Roma, 1965).

34. - y R. E. Brumberg, "Utility Analysis and Aggregate Consumption Functions", mimeografiado, 1953.

35. - "Utility Analysis and the Consumption Function: An Interpretation of Cross-Section Data", en K. Kurihara (compilador), Post-Keynesian Econo mics, New Brunswick, 1954.

36. Cynthia Taft Morris, "Intermational Comparison of Domestic Savings Rates", Washington, U. S. Agency for International Development, noviembre de 1963 pp. $1-36$

37. Population Trends in Eastern Europe, the USSR and Mainland China, Milbank Memorial Fund, Nueva York, 1960.

38. F. L. Pryor y G. Staller, "Dollar Value of the Gross National Product in Eastern Europe", Economics of Planning, febrero de 1966, 6.

39. T. Paul Schultz, "A Family Planning Hypothesis: Some Empirical Evidence from Puerto Rico", Memorándum RM-5405-RC/Aid, The RAND Corporation, 1967.

40. H. W. Singer, "Population and Economic Development", en International Development: Growth and Change, Nueva York, 1964.

41. J. J. Spengler, "The Population Obstacle to Economic Betterment", American Economic Review, mayo de 1951, 41.

42. W. Stolper, The Structure of the East German Economy, Cambridge, Mass., 1960.

43. Alan M. Strout, "Savings, Imports, and Capital Productivity in Developing Countries" (trabajo presentado al Congreso de la Sociedad Econométrica, Roma, 1965).

44. S. Swamy, "A Dynamic, Personal, Savings Function and Its Long-Run Implications", Review of Economic Statistics, febrero de 1968, 50, pp. 111-116.

45. Naciones Unidas, Demographic Yearbook, 1965, Nueva York, 1966.

46. Naciones Unidas, Comisión Económica para Europa, Some Factors in Economic Growth in Europe During the 1950's, Ginebra, 1964.

47. Naciones Unidas, CEPAL, Estudio económico de América Latina, 1963, Nueva York, 1965.

48. Naciones Unidas, "The Financing of Economic Development", World Ecomomic Survey, 1965, Parte I, Nueva York, 1966

49. Naciones Unidas, Yearbook of National Income Accormts Statistics, 1965, Nueva York, 1966.

50. U. S. Agency for International Development, Gross National Product: Growth Rates and Trend Data, marzo de 1967

51. Charles Wolf, Jr., 'Savings Regressions, 'Self-Hlelp' and Development Performance", mimeografiado, The RAND Corporation, Santa Mónica, 1964, pp. 1-28.

Traducción: Adalberto García Rocha 